\title{
Quality-of-Service Dependent Traffic Regulation for Multimedia Networks
}

\author{
Chia-Sheng Chang, Kwang-Cheng Chen \\ Institute of Communications Engineering, College of Electrical Engineering \\ National Taiwan University, Taipei, Taiwan, 10617, R.O.C. \\ E-mail: changcs@fcom.ee.ntu.edu.tw, chenkc@cc.ee.ntu.edu.tw
}

\begin{abstract}
In multimedia networks, certain applications (e.g., voice and video sessions) can tolerate some delay violations and packet losses. For these applications, in [2] we proposed a novel, more flexible traffic characterization approach- $L^{p}$-sense constraint functions - as a generalization of deterministic constraint function. Intuitively, feeding more packets than provided resources will degrade the Quality-of-Service (QoS) of multimedia applications. However, if we can control a multimedia application's output to conform to constraint functions in $L^{p}$ sense, then the portion of time prone to performance-bound violations has a normalized tail bound decaying with $x^{-p}$.

With the notion of $L^{p}$-sense traffic characterization, we investigate algorithms to shape an arbitrary arrival trace to conform to a given function $f$ in $L^{p}$ sense, i.e., $L^{p}$-sense regulation algorithms. It can be shown that under certain conditions $L^{p}$-sense regulation departure times converge to deterministic regulation departure times when $p \rightarrow \infty$. Hence $L^{p}$-sense regulators can be regarded as generalizations of the continuous-time optimal deterministic regulators discussed in [3]. By properly choosing $p$, more packets of a multimedia source can pass through $L^{p}$-sense regulators while its normalized bound-violation ratio decays fast enough to meet its QoS requirements. The basic "leaky bucket" type $L^{p}$-sense regulators are also studied as an illustration.
\end{abstract}

\section{INTRODUCTION}

Conventional deterministic regulators allow a packet to pass through only if the resulting departure process conforms to the assigned constraint function $f$ in deterministic sense (see e.g., [4]). That is, if $T[t-\tau, t$ ) (bits) denotes the amount of departure from a deterministic regulator, then $T[t-\tau, t) \leq f(\tau)$ for all $t, \tau \geq 0$. If sufficient network resources corresponding to $f$ are pre-allocated (see e.g., [5]), this implies that deterministic regulators allow a packet to pass through only if they can make sure this packet will not violate the delay bound or be discarded at intermediate network nodes. However, certain multimedia applications (e.g., voice and video sessions) can tolerate some delay violations and packet losses. For these applications, in [2] we proposed a novel, more flexible traffic characterization approach $-L^{p}$-sense constraint functions-as a generalization of deterministic constraint functions. By definition in [2] for $1 \leq p<\infty$ we say a traffic source active in $[0, \varphi]$ conforms to constraint function $f$ in $L^{p}$ sense if

$$
\frac{\|T[t-\tau, t)\|_{p}}{\left[\left.[0, \varphi]\right|^{1 / p}\right.} \triangleq \frac{\left(\int_{[0, \varphi]} T[t-\tau, t)^{p}\right)^{1 / p}}{\varphi^{1 / p}} \leq f(\tau)
$$

This research was supported by the Ministry of Education, Tai wan, R.O.C. under the contract 89-E-FA06-2-4, and by the National Science Council of Taiwan, R.O.C. under the contract NSC-89-2213E-002-120. for $0 \leq \tau \leq \varphi$, where the notation $|\cdot|$ is Lebesgue measure defined on Lebesgue measurable subsets of $\mathbb{R}[1]$. If a regulator's output conforms to constraint function $f$ in $L^{p}$ sense, it has been shown that for $0 \leq \tau \leq \varphi$

$$
\frac{|\{t \in[0, \varphi]: T[t-\tau, t) \geq \phi \cdot f(\tau)\}|}{|[0, \varphi]|} \leq \min \left\{1, \frac{1}{\phi^{p}}\right\},
$$

which is called the "normalized tail bound". Intuitively, feeding more packets than provided resources will degrade the QoS of multimedia applications. However, as long as we can guarantee the portion of time prone to delay violations or packet losses decays fast enough to satisfy the application's QoS requirements, we should try to let more packets of a multimedia application to pass through the regulator to increase transmission throughput and reduce overall delay. This is exactly the primary motivation behind our QoS dependent traffic regulators $-L^{p}$-sense traffic regulators.

\section{Basic Form of $L^{p}$-Sense Regulators}

Throughout this paper, we have the following assumptions and notations:

- $L_{\max }$ (bits) is the maximal packet size of input sources for regulation.

- For any $t \geq 0$, there are only finite number of packet arrivals in $[0, t]$.

- Given a monotone increasing real function $f:[0, \infty) \rightarrow$ $[0, \infty)$. We define $f^{-1}(x) \triangleq \inf \{s \geq 0: x \leq f(s)\}$.

- The collection of monotone increasing and leftcontinuous real functions $f:[0, \infty) \rightarrow[0, \infty)$ with $f(0)=0$ is denoted as $\mathfrak{F}$.

- A real function $f:[0, \infty) \rightarrow[0, \infty)$ is sub-additive if $f(x+$ $y) \leq f(x)+f(y)$ for all $x, y \geq 0$.

- Given a $f \in \mathfrak{F}$, we define $\bar{f}(\tau) \triangleq \sup \{g(\tau): g \in \mathfrak{F}, g \leq$ $f, g$ is sub-additive $\}$, which is called the maximal subadditive function embedded in $f$. It has been shown in [3] that a traffic source deterministically conforms to $f \in \mathfrak{F}$ if and only if it deterministically conforms to $\bar{f}$.

Given an arrival process, let $R[t-\tau, t$ ) (bits) be the amount of arrival in the interval $[t-\tau, t)$. For the convenience of our presentation, $R[t-\tau, t)$ and $T[t-\tau, t)$ are often written as $R_{\tau}(t)$ and $T_{\tau}(t)$, respectively.

We also denote the arrival time and packet length of the $n^{\text {th }}$ packet by $a_{n}$ and $L_{n}$, respectively, and an arrival process is written as a sequence of ordered pairs $\left\{\left(a_{n}, L_{n}\right)\right\}_{n=1}^{\infty}$. 
Before giving the definition of $L^{p}$-sense regulators, we need the definition of continuous-time optimal deterministic regulators [3].

Definition 1: Suppose the departure time of the $n^{\text {th }}$ packet from a continuous-time deterministic regulator $f \in$ $\mathfrak{F}$ with $f(0+) \geq L_{\max }$ (where the ' + ' means the limit from the right) is denoted by $c_{n}$. Then $c_{1}=a_{1}$ and $c_{n}=\max \left\{a_{n}, c_{n}^{\prime}\right\}$, where

$$
c_{n}{ }^{\prime}=\max \left\{c_{i}+\bar{f}^{-1}\left(\sum_{j=i}^{n} L_{j}\right): 1 \leq i \leq n-1\right\} .
$$

Now we can define a $L^{p}$-sense regulator with constraint function $f$ as follows.

Definition 2: Given $p \in[1, \infty)$, a finite time interval $[0, \varphi]$, and a monotone increasing real function $f:[0, \infty) \rightarrow$ $[0, \infty)$ with $f(0+) \geq L_{\max }$. Let

$$
g(\tau) \triangleq \begin{cases}0 & t=0 \\ f(\tau-) & t>0\end{cases}
$$

Then $g \in \mathfrak{F}$ and $g(0+) \geq L_{\max }$. Let $c_{n}$ be the $n^{\text {th }}$ departure times from the deterministic regulator with $f$.

For a fixed $s>0$ we denote by $T^{\prime}[t-\tau, t)$ the amount of departures of the output process

$$
\left\{\left(\max \left\{a_{n}, c_{n}-(n-1) s\right\}, L_{n}\right)\right\}_{n}
$$

in $[t-\tau, t)$. We set $s_{p}$, which is called the time advance value corresponding to $p$, to the least upper bound of those $s$ satisfying

$$
\frac{\left\|T^{\prime}[t-\tau, t)\right\|_{p}}{|[0, \varphi]|^{1 / p}} \triangleq \frac{\left(\int_{[0, \varphi]} T^{\prime}[t-\tau, t)^{p}\right)^{1 / p}}{\varphi^{1 / p}} \leq f(\tau)
$$

for all $0 \leq \tau \leq \varphi$. Then the $n^{\text {th }}$ departure time from this $L^{p}$-sense regulator $f$ is defined as

$$
\begin{aligned}
& b_{1}=a_{1} \\
& b_{n}=\max \left\{a_{n}, c_{n}-(n-1) s_{p}\right\} .
\end{aligned}
$$

It can be seen from the definition that $L^{p}$-sense regulation departure times are always smaller than or equal to deterministic regulation departure times. Also, as will be shown later, these $L^{p}$-sense regulators can be regarded as generalizations of optimal continuous-time deterministic regulators.

\section{Basic Properties of $L^{p}$-Sense Regulators}

The next two theorems show some properties of $L^{p}$-sense regulators.

Theorem 1: Suppose $1 \leq p_{1}<p_{2}<\infty$. If we denote by $b_{n}$ and $b_{n}{ }^{\prime}$ the $n^{\text {th }}$ departure times from a $L^{p_{1}}$-sense regulator with constraint function $f$ and $a L^{p_{2}}$-sense regulator with constraint function $f$, respectively. Then $b_{n} \leq b_{n}{ }^{\prime}$.
Proof: Fix a $s>0$ satisfies

$$
\frac{\left\|T^{\prime}[t-\tau, t)\right\|_{p_{2}}}{\|\left.[0, \varphi]\right|^{1 / p_{2}}} \triangleq \frac{\left(\int_{[0, \varphi]} T^{\prime}[t-\tau, t)^{p_{2}}\right)^{1 / p_{2}}}{\varphi^{1 / p_{2}}} \leq f(\tau)
$$

for all $0 \leq \tau \leq \varphi$. Since it is already known that

$$
\frac{\left\|T^{\prime}[t-\tau, t)\right\|_{p_{1}}}{|[0, \varphi]|^{1 / p_{1}}} \leq \frac{\left\|T^{\prime}[t-\tau, t)\right\|_{p_{2}}}{|[0, \varphi]|^{1 / p_{2}}}
$$

for $1 \leq p_{1}<p_{2}<\infty$, it follows that $s$ also satisfies

$$
\frac{\left\|T^{\prime}[t-\tau, t)\right\|_{p_{1}}}{|[0, \varphi]|^{1 / p_{1}}} \triangleq \frac{\left(\int_{[0, \varphi]} T^{\prime}[t-\tau, t)^{p_{1}}\right)^{1 / p_{1}}}{\varphi^{1 / p_{1}}} \leq f(\tau)
$$

for all $0 \leq \tau \leq \varphi$. This result implies that

$$
\{s>0: s \text { satisfies }(2)\} \subset\{s>0: s \text { satisfies }(3)\} \text {, }
$$

and consequently $s_{p_{1}} \triangleq \sup \{s>0: s$ satisfies $(3)\} \geq$ $\sup \{s>0: s$ satisfies $(2)\} \triangleq s_{p_{2}}$. Therefore it can be seen that for $n \in \mathbb{N}$

$$
\begin{aligned}
b_{n} & \triangleq \max \left\{a_{n}, c_{n}-s_{p_{1}}\right\} \\
& \leq \max \left\{a_{n}, c_{n}-s_{p_{2}}\right\} \\
& \triangleq b_{n}{ }^{\prime} .
\end{aligned}
$$

Theorem 2: Given sub-additive $f \in \mathfrak{F}$ with $f(0+) \geq$ $L_{\max }$, and an arrival process $\left\{\left(a_{n}, L_{n}\right)\right\}$. Let $b_{n}$ and $c_{n}$ be the $n^{\text {th }}$ departure times from a $L^{p}$-sense regulator with constraint function $f$ and a continuous-time deterministic regulator with constraint function $f$, respectively. Then

$$
b_{n} \rightarrow c_{n} \text { as } p \rightarrow \infty \text {. }
$$

Proof: From our assumptions, there are only a finite number of arrivals in a finite interval, and we denote this number by $N$. By definition, for a fixed $p$ we have

$$
\begin{aligned}
c_{n}-b_{n} & \leq(n-1) s_{p} \\
& \leq(N-1) s_{p} .
\end{aligned}
$$

If we can prove $s_{p} \rightarrow 0$ as $p \rightarrow \infty$, the conclusion is proved immediately.

To show $s_{p} \rightarrow 0$ as $p \rightarrow \infty$, first note that $c_{n} \geq a_{n}$ for $1 \leq n \leq N$. Suppose the $\hat{n}^{\text {th }}$ packet is the first one that its departure time from the deterministic regulator is strictly larger than its arrival time, that is

$$
\begin{aligned}
& c_{n}=b_{n}=a_{n} \text { for } 1 \leq n<\hat{n} \\
& c_{\hat{n}}>a_{\hat{n}}
\end{aligned}
$$

Suppose the $\hat{n}^{t h}$ packet departs at $t=c_{\hat{n}}-\epsilon$ for a fixed $\epsilon<c_{\hat{n}}-a_{\hat{n}}$. There exists at least one $m \in\{1, \cdots, \hat{n}-1\}$ 
such that $b_{m}=c_{m}$ and

$$
\begin{gathered}
c_{\hat{n}}-\epsilon<c_{\hat{n}}-\epsilon / 2<c_{m}+\bar{f}^{-1}\left(\sum_{j=m}^{\hat{n}} L_{j}\right) \\
=b_{m}+f^{-1}\left(\sum_{j=m}^{\hat{n}} L_{j}\right) .
\end{gathered}
$$

Therefore, it follows that

$$
T\left[t-\left(c_{\hat{n}}-b_{m}-\epsilon / 2\right), t\right) \geq \sum_{j=m}^{\hat{n}} L_{j}>f\left(c_{\hat{n}}-b_{m}-\epsilon / 2\right)
$$

for $t \in\left(c_{\hat{n}}-\epsilon, c_{\hat{n}}-\epsilon / 2\right)$ of positive measure. However, it is known that

$$
\begin{aligned}
& \frac{\left\|T^{\prime}\left[t-\left(c_{\hat{n}}-b_{m}-\epsilon / 2\right), t\right)\right\|_{p}}{|[0, \varphi]|^{1 / p}} \\
\rightarrow & \sup \left\{T^{\prime}\left[t-\left(c_{\hat{n}}-b_{m}-\epsilon / 2\right), t\right): t \in[0, \varphi]\right\} \\
\geq & \sum_{j=m}^{\hat{n}} L_{j}>f\left(c_{\hat{n}}-b_{m}-\epsilon / 2\right)
\end{aligned}
$$

for $p \rightarrow \infty$. Thus we can find $A>0$ such that

$$
\frac{\left\|T^{\prime}\left[t-\left(c_{\hat{n}}-b_{m}-\epsilon / 2\right), t\right)\right\|_{p}}{\|\left.[0, \varphi]\right|^{1 / p}}>f\left(c_{\hat{n}}-b_{m}-\epsilon / 2\right)
$$

for $p>A$, which implies

$$
s_{p}<\epsilon \text { for } p>A \text {. }
$$

Consequently, we have shown $s_{p} \rightarrow 0$ as $p \rightarrow \infty$ and proved the theorem.

In summary, Theorem 1 means that packets depart earlier from $L^{p}$-sense regulators if $p$ is smaller. The definition of $L^{p}$-sense regulators implies packet departure times from $L^{p}$-sense regulators are always earlier than that from deterministic regulators. However, Theorem 2 says that for sub-additive $f \in \mathfrak{F}$ packet departure times from $L^{p_{-}}$ sense regulators increasingly converge to the corresponding departure times from deterministic regulators as $p \rightarrow \infty$. Consequently the proposed $L^{p}$-sense regulators can be regarded as generalizations of continuous-time deterministic regulators.

However, in order to calculate time advance values and departure times of $L^{p}$-sense regulators, we need to know all departure times from deterministic regulators in time interval $[0, \varphi]$, which depend on all arrival times in time interval $[0, \varphi]$. Consequently, the $n^{\text {th }}$ departure time from $L^{p}$-sense regulators depends on packet arrival times $a_{k}, k>n$. This implies $L^{p}$-sense regulators are not causal regulators. In order to use $L^{p}$-sense regulators in real-time applications which require causal traffic regulations, we can use a recursive configuration illustrated in Figure 1.

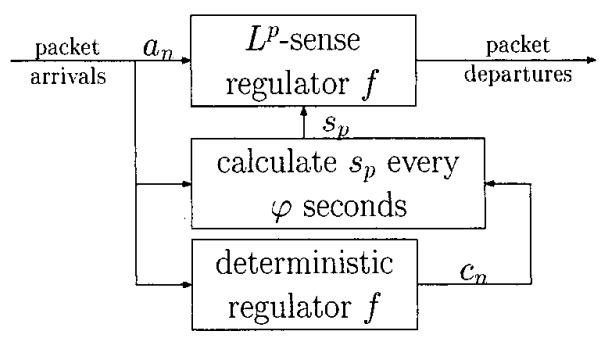

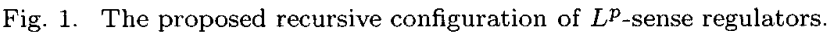

That is, the time axis is partitioned into segments of length $\varphi$. To estimate the time advance value $s_{p}$ of the next segments, in every time segment the corresponding $s_{p}$ is calculated, and the result is sent to the $L^{p}$-sense regulator. After receiving the time advance value of the previous segment, the $L^{p}$-sense regulator uses this value to regulate incoming packets that arrive in this segment. If the value of $s_{p}$ does not change too much between adjacent segments, we believe the this recursive configuration can satisfactorily produce the desirable regulation output conforming to the constraint function $f$ in $L^{p}$ sense.

\section{IV. $L^{p}$-Sense Regulators of Leaky Bucket Type}

Since leaky bucket regulators are the most basic deterministic regulators, in this section we study $L^{p}$-sense regulators of leaky bucket type, i.e., $f(\tau)=\sigma+\rho \tau$.

In order to calculate the time advance value in an interval $[0, \varphi]$, the first step is to determine a range containing it. From our assumptions, there are only a finite number of arrivals in a finite interval $[0, \varphi]$, and we denote this number by $N$. From the definition of continuous-time deterministic regulator, it can be seen that for $1 \leq n \leq N$ the $n^{\text {th }}$ departure time $c_{n}$ is always larger than or equal to the $n^{\text {th }}$ arrival time. Consider the set of real numbers

$$
\left\{\frac{c_{n}-a_{n}}{n-1}: 2 \leq n \leq N\right\} \text {. }
$$

If the this set equal to $\{0\}, s_{p}$ is set to 0 and the calculation is done. Otherwise, this set contains $M$ distinct non-zero numbers, where $M<N$. By arranging them in an increasing order, we may denote them by $e_{1}, e_{2}, \cdots, e_{M}$ and also let $e_{0}=0$. For each $e_{k}, 0 \leq k \leq M$, we check the validity of (1) with time advance value $s_{p}=e_{k}$, and then it can be found that

$$
\begin{aligned}
& \max \left\{e_{k}:(1) \text { is valid, } 0 \leq k \leq M\right\} \leq s_{p} \\
& \leq \min \left\{e_{k}:(1) \text { is invalid, } 0 \leq k \leq M\right\}
\end{aligned}
$$

For convenience, we write

$$
\begin{gathered}
\underline{s_{p}} \triangleq \max \left\{e_{k}:(1) \text { is valid, } 0 \leq k \leq M\right\} \\
\overline{s_{p}} \triangleq \min \left\{e_{k}:(1) \text { is invalid, } 0 \leq k \leq M\right\}
\end{gathered}
$$


For those packets with $c_{n}-a_{n} \leq(n-1) s_{p}$, it follows $b_{n}=a_{n}$. For those packets with $\left.c_{n}-a_{n}>\overline{(n}-1\right) s_{p}$, we have $b_{n}=c_{n}-(n-1) s_{p}$.

If we let $b_{N+1}=\varphi$, then given packet departure times $b_{1}, \ldots, b_{N}$, it can be seen that

$$
\begin{aligned}
& \int_{0}^{\varphi} T_{\tau}^{p}(t)=\int_{0}^{b_{N+1}} T_{\tau}^{p}(t) \\
= & \int_{b_{1}}^{b_{2}} T_{\tau}^{p}(t)+\int_{b_{2}}^{b_{3}} T_{\tau}^{p}(t)+\cdots+\int_{b_{N-1}}^{b_{N}} T_{\tau}^{p}(t)+\int_{b_{N}}^{b_{N+1}} T_{\tau}^{p}(t) .
\end{aligned}
$$

And each term can be written as

$$
\begin{aligned}
& \int_{b_{k-1}}^{b_{k}} T_{\tau}^{p}(t) \\
= & L_{k-1}^{p} \cdot \min \left\{\tau, b_{k}-b_{k-1}\right\}+ \\
& \left(\left(L_{k-1}+L_{k-2}\right)^{p}-L_{k-1}^{p}\right) \cdot \\
& \min \left\{\left[\tau-\left(b_{k-1}-b_{k-2}\right)\right]^{+}, b_{k}-b_{k-1}\right\} \\
& +\cdots+ \\
& \left(\left(\sum_{i=1}^{k-1} L_{i}\right)^{p}-\left(\sum_{i=2}^{k-1} L_{i}\right)^{p}\right) \cdot \\
& \min \left\{\left[\tau-\left(b_{k-1}-b_{1}\right)\right]^{+}, b_{k}-b_{k-1}\right\} \\
& k-1 \\
= & \sum_{j=1}\left(\left(\sum_{i=j}^{k-1} L_{i}\right)^{p}-\left(\sum_{i=j+1}^{k-1} L_{i}\right)\right. \\
& \min \left\{\left[\tau-\left(b_{k-1}-b_{j}\right)\right]^{+}, b_{k}-b_{k-1}\right\} .
\end{aligned}
$$

Consequently, it follows

$$
\begin{aligned}
\int_{0}^{\varphi} T_{\tau}^{p}(t)= & \sum_{k=2}^{N+1} \sum_{j=1}^{k-1}\left(\left(\sum_{i=j}^{k-1} L_{i}\right)^{p}-\left(\sum_{i=j+1}^{k-1} L_{i}\right)^{p}\right) . \\
& \min \left\{\left[\tau-\left(b_{k-1}-b_{j}\right)\right]^{+}, b_{k}-b_{k-1}\right\}
\end{aligned}
$$

which is a piecewise-linear function of $\tau$. Now consider those $\tau \in[0, \varphi]$ such that $\tau=b_{j}-b_{i}$ for some $i, j \in$ $\{1, \ldots, N+1\}$ and $i<j$, it can be seen there are only a finite number of such points. We arrange those $\tau$ in an increasing order, denote them by $\tau_{1}, \ldots, \tau_{l}$, and also let $\tau_{0}=0, \tau_{l+1}=\varphi$. Apparently, these $\tau_{j}$ are precisely the turning points of the piecewise-linear function $\int_{0}^{\varphi} T_{\tau}^{p}(t)$. Hence, for $\tau \in\left(\tau_{j}, \tau_{j+1}\right)$, it can be seen that

$$
\begin{aligned}
& \frac{d}{d \tau} \int_{0}^{\varphi} T_{\tau}^{p}(t) \\
= & \sum_{k=2}^{N+1} \sum_{j=1}^{k-1}\left(\left(\sum_{i=j}^{k-1} L_{i}\right)^{p}-\left(\sum_{i=j+1}^{k-1} L_{i}\right)^{p}\right) . \\
& \frac{d}{d \tau} \cdot \min \left\{\left[\tau-\left(b_{k-1}-b_{j}\right)\right]^{+}, b_{k}-b_{k-1}\right\} \\
= & C_{1, j},
\end{aligned}
$$

where $C_{1, j}$ is a constant depending only on $j$.

Fix a $j$ and consider those $\tau \in\left(\tau_{j}, \tau_{j+1}\right)$. Then any $s<s_{p}$ must satisfy

$$
\frac{1}{\varphi^{1 / p}}\left(\int_{0}^{\varphi} T_{\tau}^{p}(t)\right)^{1 / p} \leq f(\tau)
$$

or equivalently,

$$
\int_{0}^{\varphi} T_{\tau}^{p}(t) \leq \varphi f^{p}(\tau)
$$

for $0 \leq \tau \leq \varphi$. Using the technique of differentiation, we need to check only the boundary points $\tau_{j}, \tau_{j+1}$ and those $\tau \in\left(\tau_{j}, \tau_{j+1}\right)$ satisfying

$$
\frac{d}{d \tau}\left(\int_{0}^{\varphi} T_{\tau}^{p}(t)-\varphi f^{p}(\tau)\right)=0
$$

Suppose $f$ is of leaky bucket type, i.e., $f(\tau)=\sigma+\rho \tau$. The left hand side of the above equality can be written as

$$
C_{1, j}-\varphi p \rho(\sigma+\rho \tau)^{p-1}
$$

which has a single zero at

$$
\tau=\frac{1}{\rho}\left(\left(\frac{C_{1, j}}{\varphi p \rho}\right)^{\frac{1}{p-1}}-\sigma\right)
$$

In summary, given an arrival process $\left\{\left(a_{n}, L_{n}\right)\right\}_{n=1}^{N}$ and its deterministic regulator output process $\left\{\left(c_{n}, L_{n}\right)\right\}_{n=1}^{N}$, the time advance value $s_{p}$ is determined step by step as follows:

1. Compute $s_{p}$ and $\overline{s_{p}}$ according to (4) and (5), respectively.

2. For $1 \leq n \leq N$,

$$
b_{n}= \begin{cases}a_{n} & \text { if } c_{n}-a_{n} \leq(n-1) \underline{s_{p}} \\ c_{n}-(n-1) s & \text { if } c_{n}-a_{n}>(n-1) \underline{s_{p}}\end{cases}
$$

3. With above settings of $b_{j}$, and for each possible increasing permutation of these turning points $\left\{\tau_{j}\right\}$, find the maximal value of $s$ such that (6) holds for all $\tau=\tau_{j}$, and

(a) check if this maximal value of $s$ satisfies the corresponding increasing permutation of $\left\{\tau_{j}\right\}$.

(b) check if (6) holds for

$$
\tau=\frac{1}{\rho}\left(\left(\frac{C_{1, j}}{\varphi p \rho}\right)^{\frac{1}{p-1}}-\sigma\right), j=0, \cdots, l .
$$

If both checks are passed, this maximal value of $s$ is a candidate of time advance value $s_{p}$ corresponding to this increasing permutation of $\left\{\tau_{j}\right\}$.

4. Finally time advance value $s_{p}$ is set to the largest one of those candidate values $s$. 


\section{NumERICAL EXAMPles}

Suppose we have set up a multimedia connection with a remote friend, and have been allocated sufficient network resources corresponding to a leaky bucket function $f(\tau)=10+2 \tau$ bits (see [5]). We know this multimedia application can tolerate some packet losses due to backlogbound violations in the form that "the portion of time with backlog larger than $M \times[$ backlog bound $] \leq \max \left\{1, \frac{1}{M^{10}}\right\}$ ".

With this QoS requirements, we plan to use a $L^{10}$-sense regulator to reduce packet delay suffered in the regulation stage to get more prompt interaction.

Suppose the ratio is checked every 15 seconds $(\varphi=15)$, and we have arrival process $\left\{\left(a_{n}, L_{n}\right)\right\}=$ $\{(3,6),(5,10),(12,8),(13,7)\}$.

By feeding $\left\{\left(a_{n}, L_{n}\right)\right\}$ into a continuous-time deterministic regulator with constraint function $f$, we obtain deterministic regulation output process $\left\{\left(c_{n}, L_{n}\right)\right\}=$ $\{(3,6),(6,10),(12,8),(14.5,7)\}$.

In this example, our goal is to obtain the time advance value $s_{p}$ of a $L^{10}$-sense regulator with leaky-bucket type constraint function $f=10+2 \tau$. From $\left\{\left(a_{n}, L_{n}\right)\right\}$ and $\left\{\left(c_{n}, L_{n}\right)\right\}$, it follows that

$$
\begin{aligned}
\left\{e_{n}\right\} & =\left\{\frac{6-5}{1}, \frac{14.5-13}{3}\right\} \\
& =\{0.5,1\} .
\end{aligned}
$$

However, letting $s_{p}=1$ we find the inequality (6) is satisfied for all $\tau_{j}$ and those $\tau$ satisfying (7). This implies $s_{p}=1$. Since $s_{p} \geq s_{p}$, we must have $b_{n}=a_{n}$ for $\frac{p}{n}=1,2,3,4$, and the $L^{\frac{1}{10}}$-sense regulator output process is $\left\{\left(b_{n}, L_{n}\right)\right\}=\{(3,6),(5,10),(12,8),(13,7)\}$. Because we know $\left\{\left(b_{n}, L_{n}\right)\right\}$ satisfies $(6)$, it follows

$$
\frac{|\{t \in[0, \varphi]: T[t-\tau, t) \geq y\}|}{|[0, \varphi]|} \leq \min \left\{1,\left(\frac{f(\tau)}{y}\right)^{10}\right\} \text {. }
$$

We plot both sides of $(8)$ for $2 \leq \tau \leq 3$ in Figure 2. It shows that there exist small subsets (in the form of intervals) of $[0, \varphi]$ such that

$$
T[t-\tau, t)>f(\tau)
$$

for $2 \leq \tau \leq 3$. However, the measures of these sets are obviously smaller than the corresponding "tail bounds" predicted by (8). Hence, for this multimedia application requiring the bound-violation "normalized tail bound" decay with $x^{-10}$, the $L^{10}$-sense regulator demonstrated in this example successfully achieves this QoS requirement while letting packets pass through with less delay.

\section{Conclusions}

With the notion of $L^{p}$-sense traffic characterization introduced in [2], we proposed an algorithm to shape an arbitrary arrival trace to conform to a given function $f$ in

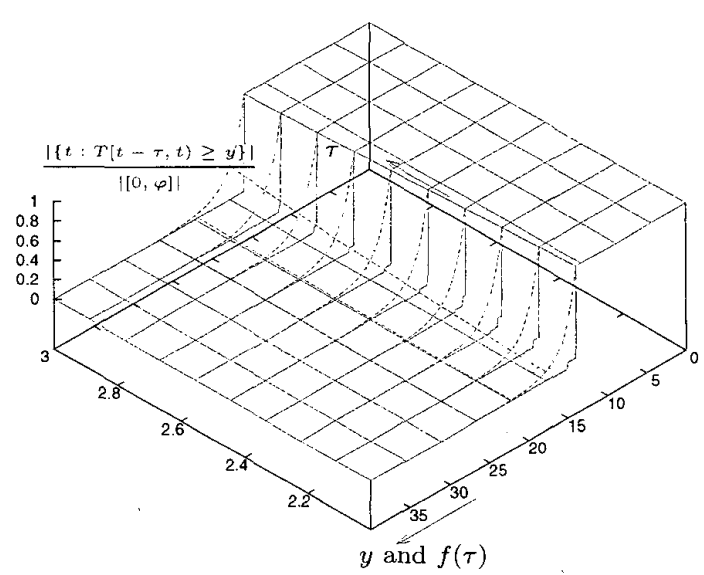

Fig. 2. Tail bound and bound-violation ratio for $\tau \in[2,3]$.

$L^{p}$-sense. The proposed algorithm has two desirable properties:

- convergence: for sub-additive, monotone increasing real function $f, L^{p}$-sense regulator departure times approach to deterministic regulator departure times as $p \rightarrow \infty$.

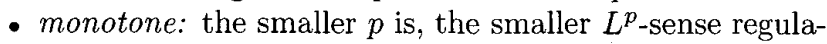
tor departure times become.

For the output process of a $L^{p_{-}}$-sense regulator, the portion of time prone to performance bound violations has been shown to have a normalized tail bound decaying with $x^{-p}$. Consequently, the parameter $p$ of a $L^{p}$-sense regulator $(1 \leq p<\infty)$ can be regarded as a measure of the strictness of regulation operation, and is very convenient to adjust to match each specific multimedia application's characteristics and QoS requirements. Compared to continuous-time deterministic regulators, $L^{p}$-sense regulators let more multimedia applications' packets to pass through according to their characteristics and QoS requirements. Therefore, these advantages and flexibilities make $L^{p}$-sense regulation a very promising regulation mechanism for future multimedia networks.

\section{REFERENCES}

[1] R. L. Wheeden, A. Zygmund, Measure and Integral: An introduction to Real Analysis, 1977.

[2] C.S. Chang, K. C. Chen; "Quality-of-Service Dependent Curve Characterization for Multimedia Networks," submitted for publication.

[3] C. S. Chang, K. C. Chen, "Continuous-Time Optimal Deterministic Traffic Regulation," submitted for publication.

[4] Cheng-Shang Chang, "On Deterministic Traffic Regulation and Service Guarantees: A systematic Approach by Filtering," IEEE Trans. on Information Theory, Vol. 44, No. 3, May 1998.

[5] C. S. Chang, K. C. Chen, "Service Curve Proportional Sharing Algorithm for Service-Guaranteed Multiaccess in Multimedia Distributed Networks," IEEE Proc. Globecom'99, Dec. 1999, Rio de Janeiro, Brazil. 